\title{
BMJ Open Prevalence of frailty in older adults in outpatient physiotherapy in an urban region in the western part of Germany: a cross-sectional study
}

\author{
Tobias Braun, ${ }^{\oplus}$ Christian Thiel, ${ }^{1,2}$ Carina Ziller, ${ }^{1}$ Julia Rasche, ${ }^{1}$ Carolin Bahns, ${ }^{1}$ \\ Lisa Happe, ${ }^{1}$ Theresa Retzmann, ${ }^{1}$ Christian Grüneberg ${ }^{1}$
}

To cite: Braun T, Thiel C, Ziller C, et al. Prevalence of frailty in older adults in outpatient physiotherapy in an urban region in the western part of Germany: a crosssectional study. BMJ Open 2019;9:e027768. doi:10.1136/ bmjopen-2018-027768

\section{- Prepublication history and} additional material for this paper are available online. To view, please visit the journal (http:// dx.doi.org/10.1136/bmjopen2018-027768).

Received 6 November 2018 Revised 11 April 2019 Accepted 12 April 2019
Check for updates

(C) Author(s) (or their employer(s)) 2019. Re-use permitted under CC BY-NC. No commercial re-use. See rights and permissions. Published by BMJ.

${ }^{1}$ Department of Applied Health Sciences, Hochschule für Gesundheit Bochum, Bochum, Germany

${ }^{2}$ Faculty of Sports Science, Ruhr University Bochum, Bochum, Germany

Correspondence to

Tobias Braun;

tobias.braun@hs-gesundheit.de

\section{ABSTRACT}

Objective To investigate the prevalence of frailty in older people in outpatient physiotherapy services in an urban region in the western part of Germany.

Design Cross-sectional study.

Setting Outpatient physiotherapy clinics were recruited in the municipal area of the city of Bochum, Germany, and selected randomly.

Participants Older adults aged 65 years and older seeking outpatient physiotherapy.

Primary and secondary outcome measures Prevalence of frailty was assessed based on the frailty phenotype model of physical frailty and the accumulation of deficit model, expressed as a Frailty Index. Prevalence was calculated for the whole sample and according to agerelated, sex-related and diagnosis-related subgroups. Results A total of 258 participants $(74 \pm 6$ years, $62 \%$ female) from 11 out of 130 (8\%) different physiotherapy clinics were included. Participants' main indication for physiotherapy was an orthopaedic or surgical condition (75\%). According to the model of a physical frailty phenotype, $17.8 \%$ (95\% Cl 13.2 to 22.5 ) participants were frail and $43.4 \%(95 \% \mathrm{Cl} 37.4$ to 49.5$)$ were prefrail. The Frailty Index identified $31.0 \%$ (95\% Cl 25.4 to 36.7 ) of individuals as frail. In both models, prevalence increased with age and was higher in women than in men. Slow gait speed $(34 \%)$, reduced muscle strength $(34 \%)$ and exhaustion $(28 \%)$ were the most prevalent indicators of physical frailty.

Conclusions Frailty is comparatively common in older patients attending physiotherapy care in Germany, with one out of three individuals being frail and every second individual being physically frail or prefrail.

Trial registration number DRKS00009384; Results.

\section{INTRODUCTION}

Most physiotherapists in industrialised countries work in outpatient physiotherapy clinics, including private practices, and care for patients with various diseases and of all ages. In 2014, 78\% of registered physiotherapists in Germany worked in outpatient clinics. ${ }^{1}$ Approximately $35 \%$ of patients prescribed for outpatient physiotherapy are 65 years and
Strengths and limitations of this study

- This study serves to enlarge the limited set of data on the epidemiology of frailty in older people referred to outpatient physiotherapy.

- A systematic approach was used to include a representative sample of community-dwelling older patients.

- The prevalence of frailty was established according to the two most common frailty models, the phenotype model of physical frailty and the accumulation of deficit model (Frailty Index).

- The study included only physiotherapy patients from one single urban region in the western part of Germany.

- The inclusion rate of physiotherapy clinics $(11 / 130=8 \%)$ was quite low.

older, ${ }^{2}$ and thus constitute one major group of patients. It has been advocated that healthcare professionals working with older people, including physiotherapists, are recommended to assess and consider the potentially higher risk state of their older clients, especially because these individuals may have special healthcare needs. ${ }^{3}$

Individuals with the same chronological age may vary widely in health and functional status. Frailty is an age-related syndrome. It describes the condition of older people's greater risk of adverse health outcomes, such as falls, institutionalisation, hospitalisation, disability and death, independently of age. ${ }^{4}$ Older people with frailty are characterised by a reduced reserve capacity of various physiological systems, so that even small stressor events can lead to significant complications or negative health outcomes. Frailty is, for example, significantly associated with a higher risk of future falls (pooled $\mathrm{OR}=1.84$, 95\% CI 1.43 to 2.38$)^{5}$ and mortality (pooled $\mathrm{HR}=2.00 ; 95 \%$ CI 1.73 to 2.32$).{ }^{6}$ 
Frailty is a consequence of cumulative decline in many physiological systems during a lifetime, and multiple etiological factors contribute to its development, including genetic and environmental factors. ${ }^{4}$ The clinical significance of frailty is increasingly recognised in older people's healthcare, and frailty is considered as one of the most challenging issues in geriatric healthcare. ${ }^{47}$

Currently, there are two major models of frailty ${ }^{47}$ : the physical frailty phenotype proposed by Fried et a ${ }^{\ominus}$ and the model of deficit accumulation proposed by Rockwood et al and Mitnitski et al. ${ }^{10}{ }^{11}$ There is no consensus on the superiority of either of these two models, but the distinction between physical frailty and the broader frailty model, which includes physical, psychological, social and mental health deficits and multimorbidity, is currently widely accepted. ${ }^{4712}$

Systematic reviews show that in industrialised countries, $\sim 14 \%$ of community-dwelling older adults have physical frailty. ${ }^{13}{ }^{14}$ Using a frailty model of deficit accumulation, the pooled prevalence of frailty is $24 \% .^{13}$ Frailty prevalence estimations vary for specific healthcare settings, such as primary care $(15 \%),{ }^{15}$ nursing homes $(19 \%-$ $76 \%)^{16}$ and emergency departments $(>50 \%) .{ }^{17}$ Frailty is more common in females than in males and prevalence increases with age. ${ }^{13} 14$ The number of frail older people in outpatient physiotherapy care is likely to be comparatively high as in the general population or in the primary care setting, but epidemiological data on frailty in physiotherapy care are limited or not available.

Information on the prevalence of frailty in outpatient physiotherapy is useful for various reasons: (1) the level of frailty may affect the outcome of rehabilitation and physiotherapy interventions. ${ }^{18}$ As older people with frailty have a decreased (physical) reserve, they may take longer to recover from conditions treated by physiotherapists, such as postsurgery and cardiovascular conditions. ${ }^{19} 20$ (2) Frailty is often unrecognised and untreated..$^{21}$ Thus, a routine screening for frailty in high-risk older populations may be beneficial to timely identify older people with increased vulnerability and corresponding medical needs. ${ }^{7}$ Such a screening can be performed by healthcare professionals, such as physiotherapists. As argued by Gustavson $e t a l,{ }^{22}$ physiotherapists 'are ideally positioned to identify frailty in geriatric healthcare settings, because they are often the first-line providers for treatment of frailty-associated functional impairments such as slowness of gait, fatigue, and weakness'. (3) Prevalence estimations of frailty in physiotherapy care can be useful for researchers to design studies on frailty in this setting.

In Germany, outpatient physiotherapy is physician directed, so patients are usually referred to outpatient physiotherapy from the general practitioner or a consulting physician, for example, a neurologist. Physiotherapy costs are usually covered by the health insurance. A typical treatment includes 6-10 sessions, which are performed in an outpatient clinic, or with special reasoning, within the patient's living environment, for example, private housing or nursing home.
This epidemiological study aimed to estimate the prevalence of frailty with regard to the conceptual model, sex, age and physiotherapy indication in community-dwelling older people receiving outpatient physiotherapy. We hypothesised prevalence estimations to be similar to those in the general population and in the primary care setting, since older people who receive outpatient physiotherapy are usually community dwelling.

\section{METHODS \\ Study design}

To exemplarily evaluate the prevalence of older people with frailty in outpatient physiotherapy services in North Rhine-Westphalia/the German Ruhr area, a prospective observational study was initiated. All participants provided written informed consent. The study protocol has been published a priori in the German Clinical Trial Register. Reporting follows the Strengthening the Reporting of Observational Studies in Epidemiology statement for the reporting of observational studies in epidemiology. ${ }^{23}$

\section{Setting}

We initially created a list of all outpatient physiotherapy clinics located in the city of Bochum, Germany. Bochum (365 000 inhabitants) is situated in the Ruhr area in North Rhine-Westphalia in the western part of Germany. The Ruhr area is the largest urban area in Germany. ${ }^{24}$ Approximately $28 \%$ of its inhabitants are $\geq 60$ years old. ${ }^{25}$

Physiotherapy clinics were searched through online address registers, web-based searches (Google) and clinic registers of the local physiotherapy association. We included all physiotherapy clinics offering outpatient physiotherapy treatments, either independently or associated with a hospital, including day clinics. If there was more than one clinic associated with the same owner or one single company, each clinic was listed and contacted separately. The search was performed independently by two researchers and it was synchronised and completed on 13 October 2015. The final list included 130 clinics.

To recruit physiotherapy clinics, an invitation letter was sent to all clinics in a randomised order by blocks of 10 clinics ('random order' function in Microsoft Excel). Non-responding clinics were additionally contacted by telephone, with a maximum of four attempts per clinic.

Clinics were screened against the following exclusion criteria via telephone: (1) <4 physiotherapists employed, regardless of the rate of employment. (2) $<20 \%$ of clients over the age of 65 years (as estimated by the clinic direction). (3) Insufficient space and facilities: to perform the study assessments in the clinic, a place and space to interview participants and to perform physical measures $(4 \mathrm{~m}$ gait speed, hand grip strengths) were required. Eligible clinics were further visited by a research assistant to discuss procedures and finally verify eligibility. The study assessment is described below in more detail. 
The study was divided into two phases: in phase I (October 2015), the first 50 clinics were contacted in randomised order. In phase II (October 2016 to April 2017), the remaining 80 clinics were contacted in randomised order.

\section{Participants}

Community-dwelling older physiotherapy patients were recruited in the eligible outpatient physiotherapy clinics. Each clinic provided a list of all patients 65 years of age and older and currently receiving 'regular' physiotherapy treatments in the clinic. We initially excluded older patients visiting the clinics for self-paid interventions or 'irregular' treatments, such as exercise training in the gym, massage sessions, hydrotherapy or any other prescriptions not covered by the public or a private health insurance. Patients solely receiving home visits were also excluded.

A research assistant screened potentially eligible patients in a face-to-face interview according to the following exclusion criteria: (1) voluntary participation, (2) language barrier (German), (3) lack of time to participate in the study examination, (4) inability to understand the study information and to give written informed consent (eg, due to severe aphasia, severe cognitive impairment/dementia or blindness) and (5) acute illness or infection.

\section{Procedures}

Eligible participants were assessed in a 15 min session either immediately before or after a regular physiotherapy treatment. To provide as much confidentiality as possible, assessments were performed in a treatment room. Only if room capacity was lacking, the assessments were performed in the waiting area and/or the corridor.

\section{Variables}

The following variables were assessed: date of birth/age, sex, body height in $\mathrm{cm}$, body weight in $\mathrm{kg}$, body mass index (BMI; $\mathrm{kg} / \mathrm{m}^{2}$ ), highest level of education, current living situation, number of medication prescribed per day, self-rated health (poor, fair, good, very good, excellent), Preferred Reporting Items for Systematic Reviews and Meta-Analyses (PRISMA)-7 screening and the presence of nine self-reported chronic diseases: high blood pressure, history of heart attack, chronic heart failure, diabetes mellitus, osteoarthritis/arthritis, history of cancer, chronic lung disease, Parkinson's disease and history of stroke.

The independent variables related to frailty, namely comorbidity, disability and polypharmacy, were rated dichotomously according to the following criteria.

\section{Comorbidity}

Comorbidity was present if two or more of the nine chronic diseases listed above were reported. ${ }^{26}$ If a condition was suspect or if diagnosis was unclear, it was not counted for comorbidity.

\section{Disability}

Disability was present if a participant reported needing help from another person to perform $\geq 1$ of the following 10 self-care tasks (basic activities of daily living) assessed with the Frailty Index ${ }^{26}$ : bathing, grooming, using the toilet, dressing, chair transfer, walking around house, stair climbing, eating, household and preparing meals.

\section{Polypharmacy}

The number of medications through which polypharmacy is 'diagnosed' varies widely in the published literature. ${ }^{27}$ In the present study, polypharmacy was indicated if a participant reported a daily intake of $\geq 5$ different medications. ${ }^{28}$ Medication was defined as any regularly prescribed item requiring a physician's order, regardless of route and including vitamins and supplements.

\section{Measurements}

Frailty was assessed according to the two common models, namely, Fried's model of a physical frailty phenotype and Rockwood and Mitnitski's model of deficit accumulation, expressed in a Frailty Index..$^{9} 10$

\section{Physical frailty phenotype}

The five domains of the physical frailty phenotype were assessed as described in the original Cardiovascular Health Study using the same cut-off points. ${ }^{9}$ Individuals with 0 deficits are considered fit/robust, those with 1 or 2 deficits are considered prefrail (intermediate) and those with $\geq 3$ deficits are considered frail.

For the grip strength item, a JAMAR dynamometer (Patterson Medical, Model 5030J1) was used following the assessment protocol proposed by Roberts $e t a .^{29}$ The average score in the dominant hand (three trials) was considered the final score. Usual gait speed (one trial, walking aids allowed) was assessed over $15 \mathrm{ft}(4.57 \mathrm{~m})$ using an additional acceleration and deceleration distance of $1 \mathrm{~m}$ each. Physical activity was assessed with the 18-item short version of the Minnesota Leisure Time Activity questionnaire $^{30}$ using a published, but not cross-culturally validated German translation. ${ }^{31}$ The outcome is kcal/ week, coded as described for the original physical frailty phenotype. ${ }^{9}$ Weight lost was scored positive if the participant had lost $\geq 10$ pounds unintentionally within the prior year. ${ }^{9}$ Self-reported exhaustion was identified by two questions from the Centre for Epidemiological StudiesDepression scale. ${ }^{9}$ If any one or two of those five variables was not assessable (eg, patient not able to walk), a deficit was considered (imputation of 1). Participants with valid data on less than three variables were excluded from the analyses.

\section{Frailty Index}

To obtain a Frailty Index, a set of selected health-related variables is recorded. In this study, a 41-item Frailty Index was calculated on the basis of the variables proposed by Searle et al. ${ }^{32}$ The score of the Frailty Index is the ratio of health deficits present to the total number of health-related variables. Thus, a 
participant with no missing measurements and 10 deficits out of 41 variables would be given a Frailty Index score of $10 / 41=0.24$. The empirical cut-off to dichotomise individuals as frail or non-frail was $\geq 0.25 .^{11}$ Thus, participants with a Frailty Index $\leq 0.24$ were considered non-frail, and a score $\geq 0.25$ indicated frailty according to the model of deficit accumulation.

To include as many participants as possible, the assessment session needed to be short and feasible, without much special equipment. Thus, we modified the Frailty Index used by Searle $e t a l^{32}$ by excluding, adding and changing some variables. All included variables satisfied the criteria proposed by Searle et al. ${ }^{32}$ : (1) health-related, (2) age-associated, (3) neither overly common (deficit was present in $80 \%$ or more of individuals by age 80 years) and (4) nor overly uncommon (present in $<1 \%$ of the study population). The Frailty Index used in this study, together with a detailed description of all alterations from the version proposed by Searle $e t$ $a l^{32}$ is presented in table 1 and the descriptions below, respectively.

We deleted the variable 'help lifting $10 \mathrm{lbs}$ ' because this item was evaluated as infeasible in our pilot assessments.

The 'peak flow' and 'shoulder strength' measures were $e x$ cluded because of feasibility reasons.

The variable 'cut down on usual activity (in the last month)' was replaced by item \#3 from PRISMA-7: 'In general, do you have any health problems that require you to limit your activities?' because this question assesses the same deficit (activity restrictions). The same coding was used: yes $=1 ;$ no $=0$.

The variable 'have trouble getting going', which is intended to assess depressive mood, was replaced by the self-reported use of antidepressive medication within the last month and coded as yes $=1$, suspect $=0.5$ and no $=0$.

The cognition deficit, originally assessed with the Mini-Mental State Examination test, was replaced by the question 'Do you think that you have more problems with your memory than most other people?', as used by others, ${ }^{33}$ and coded as yes $=1$ or no $=0$.

We added the following four items from PRISMA-7, all coded as yes $=1$ or no $=0$ : Item \#4: 'Do you need someone to help you regularly?' (deficit of social support); item \#5: 'In general, do you have any health problems that require you to stay at home?' (deficit of health-related participation restriction); item 7: 'Do you regularly use a cane, a walker, or a wheelchair to move about?' (deficit of mobility limitation); item \#6: 'If you need help, can you count on someone close to you?' (deficit of social support) coded as yes $=0$ or no $=1$.

Grip strength and gait speed (habitual and fast) were rated based on actual physical performance. All other items were patient reported. For grip strength, the average score in the dominant hand (three trials) was considered the final score. The measurement procedure followed the recommendations by Roberts $e t a l^{29}$
PRISMA-7 QUESTIONNAIRE

PRISMA-7 is a 7-item frailty screening questionnaire. ${ }^{34}$ A score of $\geq 3$ out of 7 is considered to indicate possible frailty. The PRISMA-7 screening has been identified as the most promising self-reported screening instrument in a systematic review. ${ }^{35}$ We used a validated German version of the questionnaire. ${ }^{36}$

\section{Bias}

A relevant source of bias with respect to the generalisability of the results is the representativeness of the study sample. We assumed many older physiotherapy patients to refuse participation because of time restrictions. These people were asked to perform the short PRISMA-7 questionnaire to compare the level of frailty in the group of excluded people with that in the study population.

\section{Study size}

We intended to include at least 250 participants based on the available budget for this study.

\section{Statistical methods}

Descriptive statistics were used to describe the characteristics of the study population and the prevalence of frailty. Study participants were dichotomised into groups of 'frail' and 'non-frail' (fit/robust) individuals based on their Frailty Index or physical frailty phenotype score, respectively. Prevalence is the number of frail individuals divided by the total number of participants assessed (Prevalence $=$ number of affected individuals at the time of investigation/number of included individuals in the investigation). Only participants with complete data in both frailty measures were included. There was no imputation for missing data.

Interval-based data were examined for normal distribution with the Shapiro-Wilk test of normality and by visual inspection of the related histograms and P-P plots. Baseline characteristics of the frail and non-frail groups were compared using t-tests, Mann-Whitney $\mathrm{U}$ tests and $\chi^{2}$ tests, as appropriate.

The prevalence estimates were calculated according to both diagnostic models (physical frailty phenotype and Frailty Index) and based on the whole sample and subjected to sex-specific and age-related subgroups. ${ }^{14}$ We aimed to report the prevalence of frailty according to 5-year groups: $65-69,70-74,75-79,80-84,85-89$ and $90+$ years. However, there were no participants older than 89 years, and only 40 participants were older than 79 years. Thus, we collapsed the latter three age categories (80+ years). Prevalence was also calculated according to the medical diagnosis that indicated the physiotherapy prescription (orthopaedic/surgical, neurological or internal medicine/cardiorespiratory/cancer).

In the original study on Fried's physical frailty phenotype, ${ }^{9}$ participants with Parkinson's disease, stroke, a MiniMental score of $<18 / 30$ points and those who were taking antidepressants were excluded from the study, since 'these conditions could potentially present with frailty 
Table 1 The list of health deficit variables included in the Frailty Index and how they were coded as deficits

\begin{tabular}{lll}
\hline No & Variable & Cut point \\
\hline 1 & $\begin{array}{l}\text { In general, do you have any health problems that } \\
\text { require you to limit your activities? }\end{array}$ & Yes=1, no=0 \\
2 & $\begin{array}{l}\text { Do you need someone to help you regularly? } \\
\text { In general, do you have any health problems that } \\
\text { require you to stay at home? }\end{array}$ & $\begin{array}{l}\text { Yes }=1, \mathrm{no}=0 \\
\text { If you need help, can you count on someone close to }\end{array}$ \\
$\begin{array}{ll}\text { you? } & \text { Yes=0, no=1 }\end{array}$
\end{tabular}

5 Do you regularly use a cane, a walker or a wheelchair $Y e s=1, \mathrm{no}=0$ to move about?

$6 \quad$ Help bathing $\quad$ Yes $=1$, no $=0$

$7 \quad$ Help grooming $\quad$ Yes $=1$, no $=0$

8 Help using toilet $\quad$ Yes $=1$, no $=0$

$9 \quad$ Help dressing $\quad$ Yes $=1$, no $=0 \quad t$

10

11

12

13

14

15

16

17

18

19

20

21

22

23

24

25

26

27

28

29

30

31

32

33

34

35

35

37

38

39

40
Help getting in/out of chair

Help walking around house

Help up/down stairs

Help eating

Help shopping

Help with housework

Help with meal preparations

Help taking medication

Help with finances

Lost $>10$ lbs in last year (unintentionally)

Self-rating of health

How health has changed in last year

Do you think that you have more problems with your memory than most other people?

Stayed in bed at least half the day due to health (in $Y e s=1$, no $=0$ last month)

Walk outside (last week)

Feel everything is an effort

Feel depressed

Feel happy

Feel lonely

Antidepressive medication (last month)

High blood pressure

Heart attack

Chronic heart failure

Stroke

Cancer

Diabetes

Arthritis

Chronic lung disease

Body mass index§

Grip strength§

Usual pace§

Yes $=1, \mathrm{no}=0$

Yes $=1, \mathrm{no}=0$

Yes $=1$, no $=0$

Yes $=1, \mathrm{no}=0$

Yes $=1, \mathrm{no}=0$

Yes $=1$, no $=0$

Yes $=1$, no $=0$

Yes $=1$, no $=0$

Yes $=1$, no $=0$

Yes $=1$, no $=0$

Yes $=1, \mathrm{no}=0$
Poor $=1$, fair $=0.75$, good $=0.5$, very

good $=0.25$, excellent $=0$

Worse $=1$, better $/$ same $=0$

Origin

x

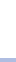

(1)

$\dagger$

$\dagger$

†

$\dagger$

$\dagger$

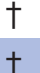

$\dagger$

$\dagger$

$+$

$\dagger$

$+$

$\dagger$

$\dagger$

$\dagger$

$+$

$\dagger$

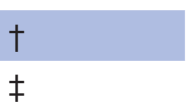

$\ddagger$

$<3$ days $=1, \geq 3$ days $=0$

$\dagger$

$\dagger$

Most of time $=1$, some time $=0.5$, rarely $=0 \dagger$

Most of time $=1$, some time $=0.5$, rarely $=0 \dagger$

Most of time $=0$, some time $=0.5$, rarely $=1 \dagger$

Most of time $=1$, some time $=0.5$, rarely $=0 \dagger$

Yes $=1$, suspect $=0.5$, no $=0$

Yes $=1$, suspect $=0.5$, no $=0 \quad \dagger$

Yes $=1$, suspect $=0.5$, no $=0 \quad \dagger$

Yes $=1$, suspect $=0.5$, no $=0 \quad \dagger$

Yes $=1$, suspect $=0.5$, no $=0 \quad \dagger$

Yes $=1$, suspect $=0.5$, no $=0 \quad \dagger$

Yes $=1$, suspect $=0.5$, no $=0 \quad \dagger$

Yes $=1$, suspect $=0.5$, no $=0 \quad \dagger$

Yes $=1$, suspect $=0.5$, no $=0 \quad \dagger$

See below $\quad \dagger$

See below $\quad+$

See below $\dagger$ 
Table 1 Continued

\begin{tabular}{|c|c|c|c|c|c|}
\hline No & Variable & & Cut point & & Origin \\
\hline 41 & Rapid pace§ & & See below & & $\dagger$ \\
\hline \multicolumn{2}{|c|}{ Variable } & Deficit for men & & Deficit for women & \\
\hline \multicolumn{2}{|c|}{ Body mass index (BMI) } & $\begin{array}{l}<18.5, \geq 30 \text { as a deficit. } \\
25-<30 \text { as a 'half deficit' }\end{array}$ & & $\begin{array}{l}<18.5, \geq 30 \text { as a deficit. } \\
25-<30 \text { as a half deficit }\end{array}$ & \\
\hline \multicolumn{2}{|c|}{$\begin{array}{l}\text { Grip strength (GS in kg; dominant hand; } \\
\text { mean of three trials) }\end{array}$} & $\begin{array}{l}\text { For } \mathrm{BMI} \leq 24, \mathrm{GS} \leq 29 \\
\text { For } \mathrm{BMI} 24.1-28, \mathrm{GS} \leq 30 \\
\text { For } \mathrm{BMI}>28, \mathrm{GS} \leq 32\end{array}$ & & $\begin{array}{l}\text { For } \mathrm{BMI} \leq 23, \mathrm{GS} \leq 17 \\
\text { For } \mathrm{BMI} 23.1-26, \mathrm{GS} \leq 17.3 \\
\text { For } \mathrm{BMI} 26.1-29, \mathrm{GS} \leq 18 \\
\text { For } \mathrm{BMI}>29, \mathrm{GS} \leq 21\end{array}$ & \\
\hline \multicolumn{2}{|c|}{ Rapid pace walk (s) } & $>7.5$ & & $>7.5$ & \\
\hline \multicolumn{2}{|c|}{ Usual pace walk (s) } & $>12.0$ & & $>12$ & \\
\hline
\end{tabular}

*Taken from PRISMA-7 screening.

†Taken from Searle et al. ${ }^{32}$

$\ddagger$ Taken from Abner et $a l^{33}$

$\S$ Continuous variable cut-points.

PRISMA, Preferred Reporting Items for Systematic Reviews and Meta-Analyses.

characteristics as a consequence of a single disease'. Thus, we additionally calculated the prevalence of frailty in a sample excluding participants with Parkinson's disease, a history of stroke, self-reported memory complaints and antidepressant medication (analysis not described in the study protocol).

The prevalence of participants identified as frail according to both models was also calculated.

All analyses were performed using SPSS for Windows V.24.0. The level of statistical significance was set to $\mathrm{p}<5 \%$.

\section{Patient and public involvement}

No patients were involved in the development of the research question, study design or interpretation of the data.

\section{RESULTS}

\section{Participants}

The flow of outpatient physiotherapy clinics and participants through the study is illustrated in figure 1. Participants were recruited from 11 different clinics, which constituted $8 \%$ of the 130 clinics invited to this study. The mean number of older patients currently under physiotherapy treatment per clinic was $49 \pm 17$ (range: 26-85), and the mean rate of patients recruited per clinic was $47 \% \pm 8 \%$ (range: $29 \%-59 \%$ ).

The characteristics of the 258 participants are given in table 2 . The mean age was 74 years, women accounted for $62 \%$ of the participants, $47 \%$ reported polypharmacy, $40 \%$ of the participants were disabled and $\sim 2$ out of 3 participants had comorbidity. Most participants received physiotherapy because of an orthopaedic or surgical disorder $(72 \%)$.

Fourteen of the 286 excluded patients (5\%) completed the PRISMA-7 screening. No further comparison between this group of excluded people and the study participants was performed due to the small sample size.
There were no participants excluded from the analyses due to missing data in any frailty measure. In table 2 , frail and non-frail participants are compared. In both frailty models, patients with frailty were significantly older, used more medication, reported poorer health, had less education and scored higher on the PRISMA-7 screening. The group of patients with frailty included more women, more people dependent on a walking aid, more people with memory complaints and more people with a depressive mood (all $\mathrm{p}<0.05)$. People with frailty according to the model of deficit accumulation had a higher BMI, had more comorbidities and reported a history of stroke more frequently (all $\mathrm{p}<0.05$ ). Conversely, these factors did not influence physical frailty significantly.

\section{Main results}

The prevalence of physical frailty was $17.8 \%$ (95\% CI $13.2 \%$ to $22.5 \%$ ), and $43.4 \%$ (95\% CI $37.4 \%$ to $49.5 \%$ ) of patients were considered prefrail. Using the deficit accumulation model of frailty, the prevalence was $31.0 \%(95 \%$ CI $25.4 \%$ to $36.7 \%$ ).

Prevalence rates according to both models, namely, the physical frailty phenotype and the model of deficit accumulation (Frailty Index) and according to age-related, sex-related and diagnosis-related groups are given in figures 2 and 3, respectively. In both models, prevalence increased with age and was higher in women than in men. Frailty prevalence was also higher in patients with a neurological diagnosis than in patients receiving outpatient physiotherapy services due to internal medicine or cancer-related disorders or orthopaedic/surgical diagnoses. The prevalence of prefrailty is illustrated in the figure in the online supplementary file 1 . The bar chart figure in the online supplementary file 2 illustrates the prevalence of physical prefrailty and frailty combined in one figure. 


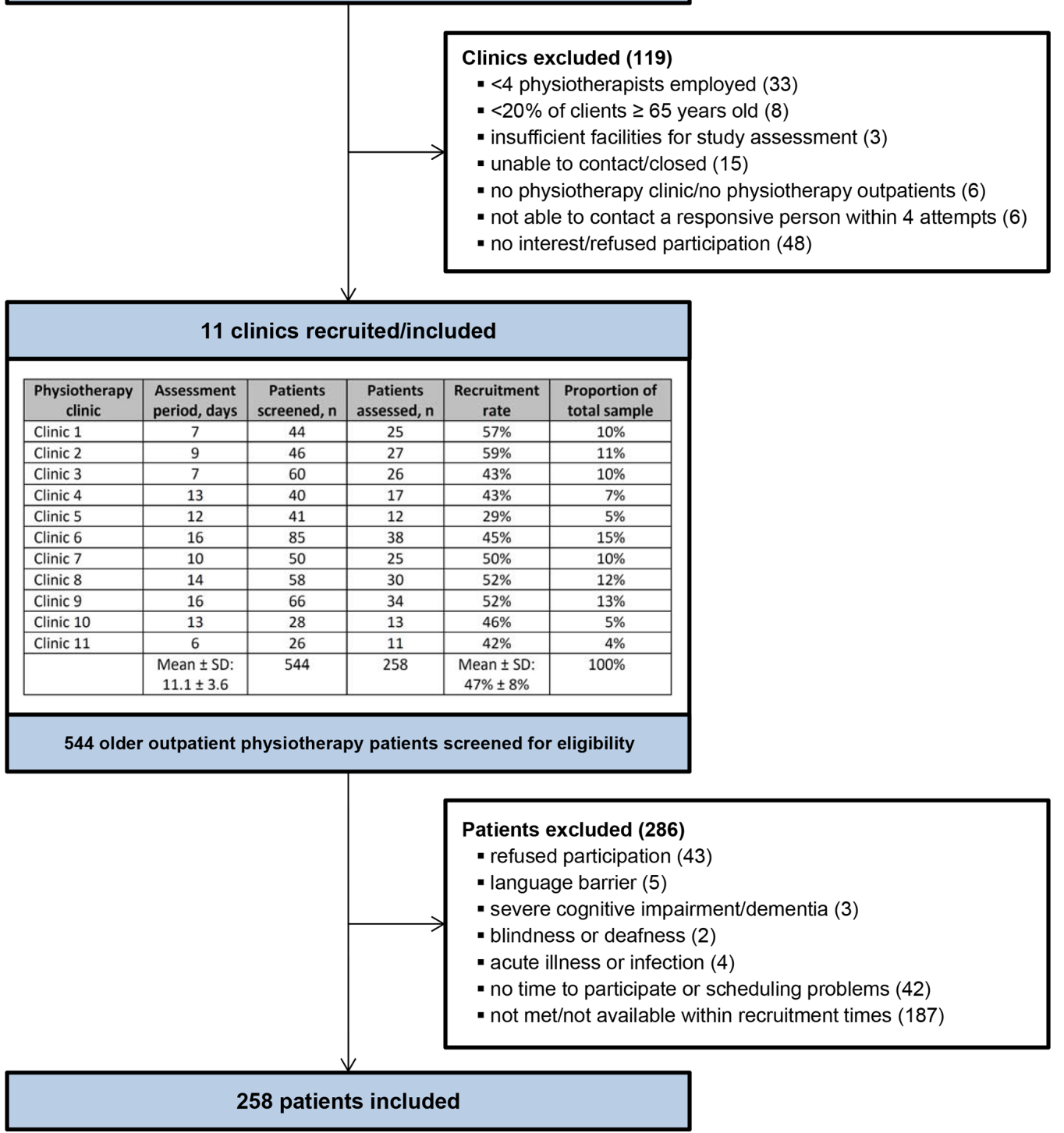

Figure 1 Flow of outpatient physiotherapy clinics and participants through the study.

Of those 100 robust participants without physical frailty, $3(3 \%, 95 \%$ CI 0 to 6.3$)$ were considered frail based on a Frailty Index $\geq 0.25$. Thirty-six of 112 physically prefrail $(32.1 \%, 95 \%$ CI 23.5 to 40.8$)$ and 41 of 46 physically frail $(89.1 \%, 95 \%$ CI 80.1 to 98.1$)$ participants were considered frail according to the model of deficit accumulation. Thus, the prevalence of participants identified as frail according to both models was $15.9 \%$ (95\% CI 11.4 to 20.4$)$.

\section{Other analyses}

When excluding participants with Parkinson's disease $(n=11)$, a history of stroke $(n=35)$, self-reported memory complaints $(\mathrm{n}=31)$ and antidepressant medication $(n=62)$, the prevalence of physical prefrailty and physical frailty of the remaining 159 participants was $38.4 \%(95 \%$ CI $30.8 \%$ to $45.9 \%$ ) and $10.7 \%$ (95\% CI $5.8 \%$ to $15.5 \%)$, respectively.
Figure 4 illustrates the rate of deficits assessed in the physical frailty phenotype (weight loss, inactivity, exhaustion, slow gait speed, weakness) of the total sample and according to subgroups of prefrail and frail participants. Slow gait speed and low grip strength were the most frequent physical deficits $(34 \%)$. Similarly, slowness and weakness were the most frequent ( $\geq 40 \%$ each) deficits in patients with prefrailty, and slowness and exhaustion were each present in $>90 \%$ of patients with physical frailty. Slowness, weakness and exhaustion were also the most prevalent $(60 \%-70 \%)$ physical frailty factors in the participants with frailty of deficit accumulation.

\section{DISCUSSION}

In this epidemiological study, the overall prevalence of frailty in older physiotherapy outpatients in the city of Bochum in Germany was $17.8 \%$ using Fried's model of 


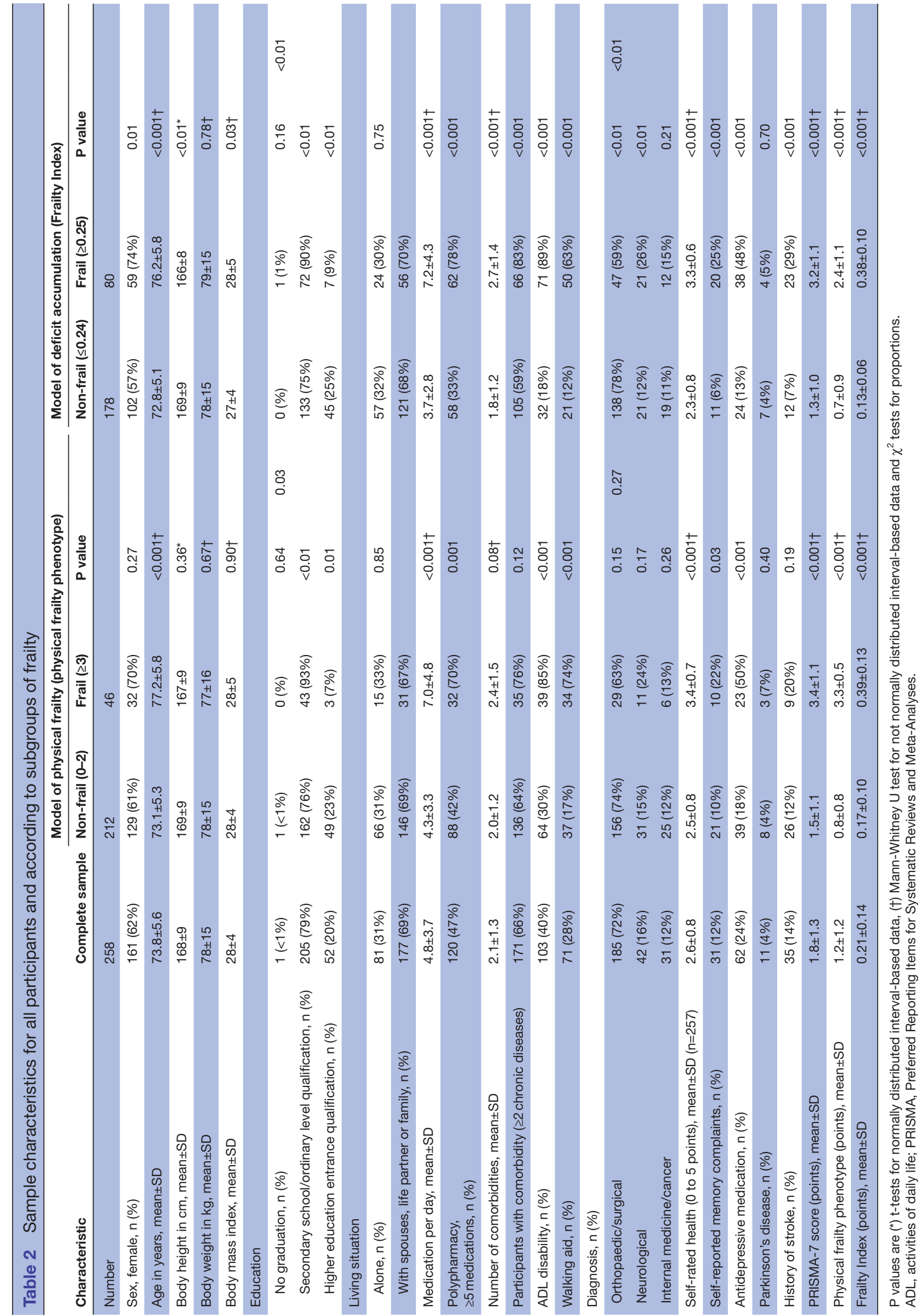




\section{n Prevalence (95\% confidence interval)}

Complete sample

Age groups (years)

65-69

70-74

75-79

$80+$

Gender

Women

Men

Physiotherapy indication

Orthopaedic/surgical

Neurological

Internal medicine/cancer
$25817.8 \%(13.2 \%-22.5 \%)$

$72 \quad 11.1 \%(3.9 \%-18.4 \%)$

$70 \quad 7.1 \%(1.1 \%-13.2 \%)$

$76 \quad 19.7 \%(10.8 \%-28.7 \%)$

$40 \quad 45.0 \%(29.6 \%-60.4 \%)$

$16119.9 \%(13.7 \%-26.0 \%)$

$97 \quad 14.4 \%(7.4 \%-24.4 \%)$

$18515.7 \%(10.4 \%-20.9 \%)$

$42 \quad 26.2 \%(12.9 \%-39.5 \%)$

$31 \quad 19.4 \%(5.4 \%-33.3 \%)$
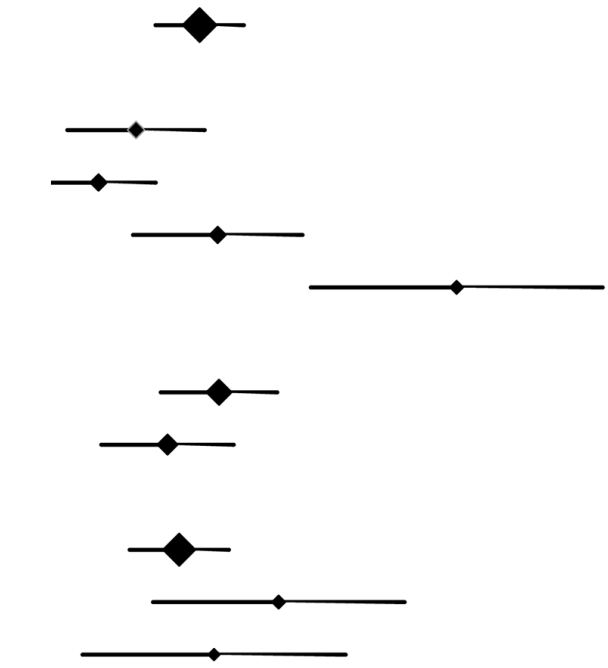

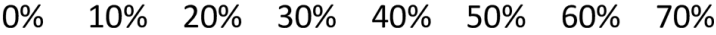

Figure 2 The prevalence of physical frailty (Fried's model of a physical frailty phenotype) with 95\% Cls for the complete sample and according to groups for sex, age and physiotherapy indication.

a physical frailty phenotype and $31.0 \%$ using a model of deficit accumulation. Physical prefrailty was present in $43.4 \%$ of participants. Frailty was more common in older individuals and in women, but the prevalence in each age and sex category differed depending on the model used.

The higher prevalence of frailty according to the model of deficit accumulation, in females and with increased age has been consistently reported in previous reviews and meta-analyses. ${ }^{13} 143738$ A possible explanation for higher prevalence estimations based on the Frailty
Index assessment is that this model also includes mental, emotional and social aspects, and chronic diseases. Our findings indicate that the prevalence of frailty was not significantly different between sexes, but tends to be higher in women than in men.

We can confirm our hypotheses that the prevalence of frailty in outpatient physiotherapy is similar to prevalence estimations for the general population. However, the prevalence of physical frailty found in this sample of physiotherapy patients $(17.8 \%, 95 \%$ CI 13.2 to 22.5$)$

\section{Complete sample \\ Age groups (years)}

64-69

70-74

75-79

$80+$

Gender

Women

Men

Physiotherapy indication

Orthopaedic/surgical

Neurological

Internal medicine/cancer n Prevalence ( $95 \%$ confidence interval)

$258 \quad 31.0 \%(25.4 \%-36.7 \%)$

$72 \quad 18.1 \%(9.2 \%-26.9 \%)$

$70 \quad 25.7 \%(15.5 \%-36.0 \%)$

$76 \quad 34.2 \%(23.5 \%-44.9 \%)$

$40 \quad 57.5 \%(42.2 \%-72.8 \%)$

$16136.6 \%(29.2 \%-44.1 \%)$

$9721.6 \%(13.5 \%-29.8 \%)$

$18525.4 \%(19.1 \%-31.7 \%)$

$42 \quad 50.0 \%(34.9 \%-65.1 \%)$

$3138.7 \%(21.6 \%-55.9 \%)$
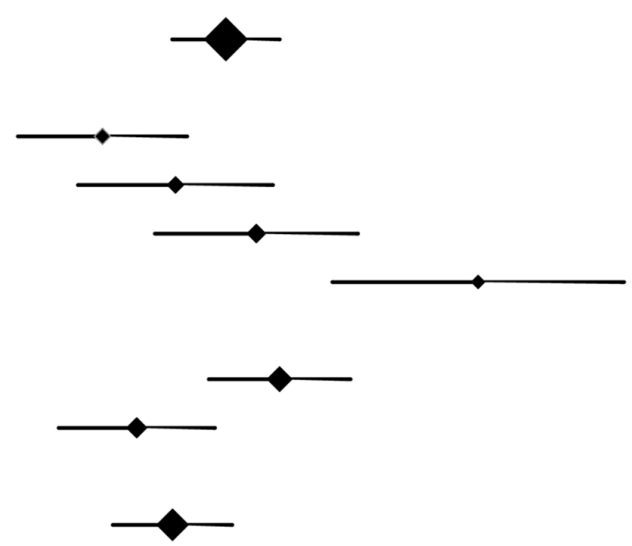

Figure 3 The prevalence of frailty (Frailty Index/model of deficit accumulation) with $95 \%$ Cls for the complete sample and according to groups for sex, age and physiotherapy indication. 

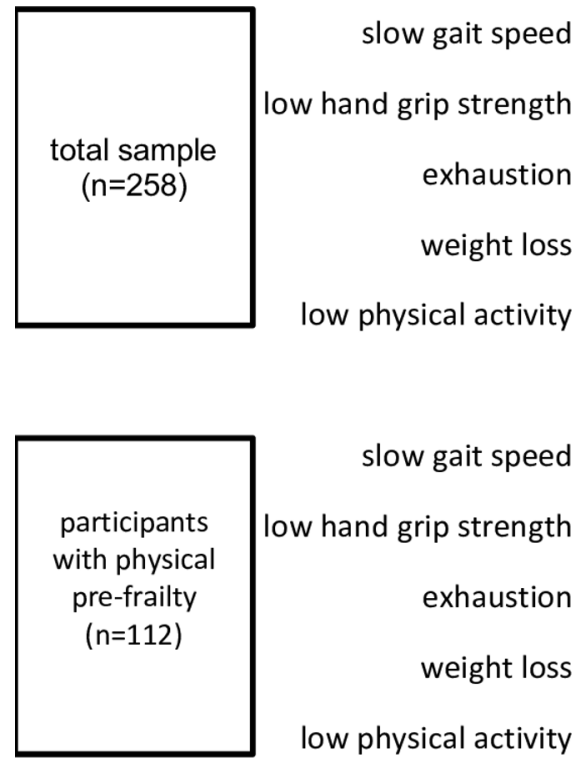

slow gait speed low hand grip strength

exhaustion

weight loss

low physical activity

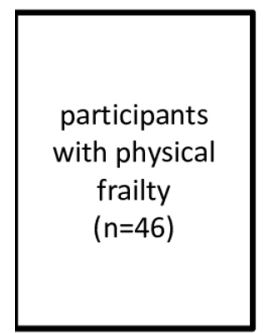

slow gait speed

low hand grip strength

exhaustion

weight loss

low physical activity

slow gait speed

particpants without frailty

of deficit

accumulation

$(n=178)$

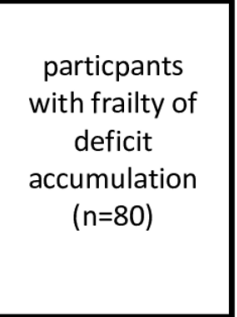

low hand grip strength

exhaustion

weight loss

low physical activity

slow gait speed

low hand grip strength

exhaustion

weight loss

low physical activity

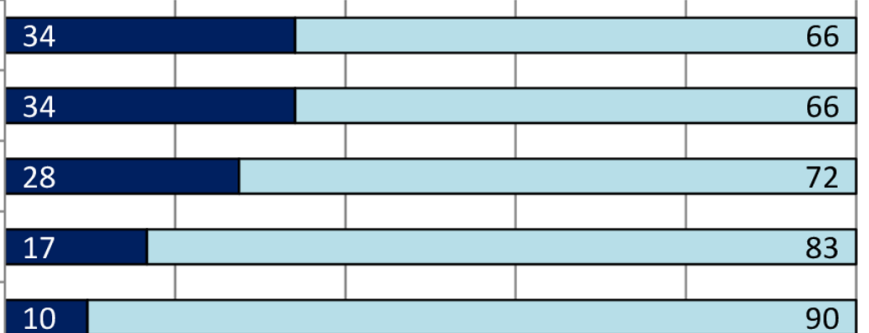

10
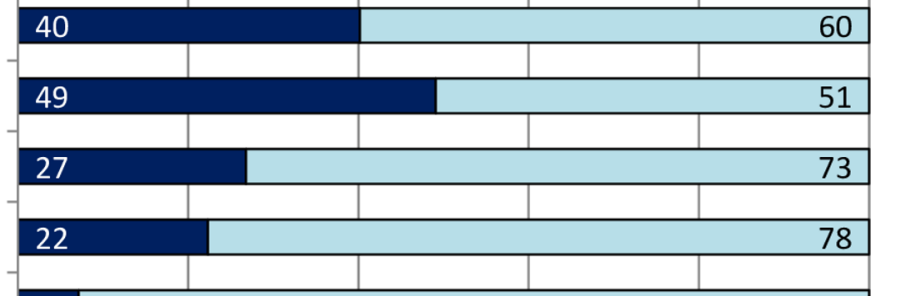

7
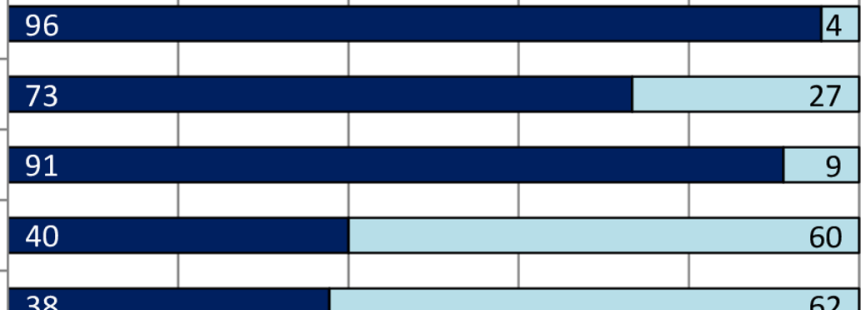

38
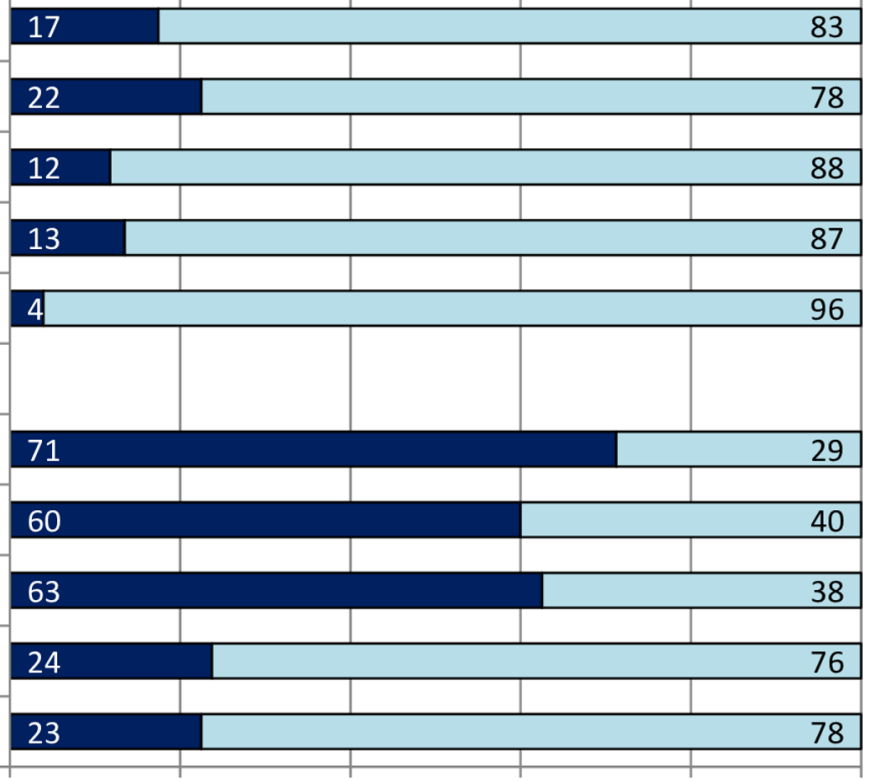

$\begin{array}{lllll}0 \% & 20 \% & 40 \% & 60 \% & 80 \%\end{array}$

Figure 4 Frequency of physical frailty phenotype deficits in the subgroups of participants.

is slightly higher compared with pooled frailty prevalence estimations reported for community-dwelling older adults of $14.4 \%$ (95\% CI 3.8 to 54.2$)^{13}$ and $9.9 \%$ (95\% CI 9.6 to 10.2). ${ }^{14}$ The prevalence of physical frailty in representative samples of older community-dwelling adults from 10 European countries is $17.0 \%$ (95\% CI 15.3 to 18.7 ), ranging from $5.8 \%$ in Switzerland to $27.3 \%$ in Spain, and $12.1 \%$ (95\% CI 8.8 to 15.3 ) for Germany. ${ }^{37}$ However, Fuchs et $a l^{39}$ used four population-based studies to describe the prevalence of frailty in Germany, and 
reported sex-stratified prevalence estimations of physical frailty from $2.5 \%$ to $11.4 \%$. Results vary due to methodological different between the studies, such as design, population and frailty assessment. For example, Buttery et $a t^{40}$ reported a lower frailty prevalence of $2.6 \%(95 \%$ CI 1.8 to 3.6) in 1843 older community-dwelling people living in Germany. The authors ${ }^{40}$ used a modified physical frailty phenotype assessment and included only participants at the age of 65-79 years, what might have resulted in the lower estimates of frailty.

A possible explanation for the higher rate of individuals with physical frailty compared with other studies might be the broader inclusion criteria of this study. In the original study on Fried's physical frailty phenotype, ${ }^{9}$ participants with Parkinson's disease, stroke, a Mini-Mental score of $<18 / 30$ points and those who were taking antidepressants were excluded from the study. In this respect, our additional analysis revealed a prevalence of physical prefrailty and physical frailty of $38.4 \%$ (95\% CI $30.8 \%$ to $45.9 \%$ ) and $10.7 \%$ (95\% CI $5.8 \%$ to $15.5 \%$ ), respectively. These results might also explain the higher prevalence of frailty in those 42 participants prescribed for physiotherapy because of a neurological disorder, including 10 people with Parkinson's disease and 23 with stroke. For these individuals, it is unclear if the clinical symptoms of the neurological disorder and/or the age-associated accelerated gradual decrease in physiological reserve and failing homoeostatic mechanisms discussed for the pathophysiological pathway of frailty contribute to a positive assessment of frailty. ${ }^{4}$

According to a Frailty Index assessment, 31.0\% (95\% CI 25.4 to 36.7) of physiotherapy patients had frailty. This figure is higher than the pooled frailty prevalence reported by Shamliyan $e t a l^{13}$ of $23.6 \%$ (95\% CI 18.0 to 30.9 ), with overlapping $95 \%$ CIs. Saum et $a t^{41}$ reported a prevalence of frailty in 2265 older adults (65-69 years) in Germany of $57.7 \%$ (Frailty Index $>0.20$ ). Using a Frailty Index cut-off score of $>0.20$, the prevalence of frailty in the present sample was $42.2 \%$ (95\% CI 36.2 to 48.3 ).

The prevalence of physical frailty in older physiotherapy outpatients is also comparable to prevalence figures reported for the primary care setting. For example, Drey et $a l^{15}$ assessed older patients seen by a general practitioner in Germany and reported a prevalence of physical frailty of $14.3 \%$ (95\% CI 8.6 to 21.9 ). Since this is the first study on the prevalence of frailty in outpatient physiotherapy, we cannot compare our data to any other data from this specific healthcare setting.

All comparisons between frailty prevalence estimations found in this study and prevalence estimations reported in systematic reviews and meta-analyses ${ }^{13} 1437$ indicate a similar, but higher prevalence of frailty in older patients in outpatient physiotherapy than in the general community-dwelling older population. A possible explanation might be the fact that the present sample included older patients prescribed for physiotherapy because of a specific (acute, subacute or chronic) medical condition that might contribute to the characteristics of frailty, according to both models. For example, older patients with acute or chronic non-specific low back pain might have reduced levels of physical activity and/or functional disabilities in daily life. ${ }^{42}$ However, we excluded patients with dementia or blindness, and patients prescribed for home visits, including home-bound older people with high levels of disability and people living in residential care settings. This procedure is likely to result in an underestimation of the true prevalence of frailty in older patients prescribed for outpatient physiotherapy.

The most prevalent indicators of physical frailty were slow gait speed (34\%), low grip strength (34\%) and exhaustion (28\%). In the Cardiovascular Health Study by Fried $e t a l,{ }^{9}$ low activity (22\%), slow walk and low grip strength (both 20\%) were the most common components of physical frailty (prevalence: 6.9\%). Santos-Eggimann et $a l^{37}$ used a modified frailty phenotype assessment and reported exhaustion (37\%), weakness (26\%) and slowness $(23 \%)$ to be the most prevalent indicators of physical frailty in community-dwelling older Europeans at the age of 65 years and older (frailty prevalence: $17.0 \%$ ). Deviations might result from the differences in sample compositions, sample sizes, study settings, countries and frailty prevalence estimations between studies.

\section{Limitations}

The generalisability of the present results is limited because we only included physiotherapy patients from one single urban region in the western part of Germany. Further studies should evaluate the prevalence of frailty in outpatient physiotherapy in other and/or more regions of Germany, and other countries, including clinics from both, urban and rural areas.

We aimed to invite and include participants from all outpatient physiotherapy clinics in the study city (Bochum). Although we put much effort in making an inventory of all existing clinics, including a broad search performed by two independent researchers, we might have missed some clinics. The inclusion rate of physiotherapy clinics $(11 / 130=8 \%)$ is quite low and might be explained by our strict inclusion and exclusion criteria. Especially the requirement of at least four employed physiotherapists led to the exclusion of 33 clinics. The most likely reasons for refusal to participate were the lack of (financial) incentives and the aim of the study. We assume less interest of physiotherapists in epidemiological research on a population of people with a rather new/uncommon condition (frailty), that is not listed in the $10^{\text {th }}$ revision of the International Statistical Classification of Diseases and Related Health Problems (ICD-10), than, for example, in an interventional study on conditions typically treated by physiotherapists, such as stroke or low back pain. However, the randomly selected 11 clinics included were located in different districts of the city and we assume a representative sample of physiotherapy clinics for a larger urban area in western Germany. Moreover, the present sample seems very similar in comparison to samples from other frailty prevalence studies in 
community-dwelling older adults with respect to the main sociodemographic characteristics such as sex distribution (62\% vs $59 \%-64 \%$ female $^{43-45}$ ), mean age (74 vs $72-75$ years $\left.^{43-45}\right)$ and BMI (28 vs $27 \mathrm{~kg} / \mathrm{m}^{2^{45}}$ ).

Concerning the sample of 258 participants, a selection bias might exist since we excluded patients prescribed for home-based physiotherapy and $53 \%$ of patients invited could not be included. We were not able to compare the group of excluded people to the study population, as planned, due to the small sample size. It is likely that especially older people and patients with higher levels of frailty might have refused participation, as reported by others. ${ }^{46}$ However, we reached the a priori defined sample size of 250 participants, which is higher than in some, ${ }^{1547}$ but lower than in most other studies on the prevalence of frailty. ${ }^{13141638}$ Future studies might use routine data of outpatient physiotherapy to better describe the prevalence of frailty in this population.

The prevalence of frailty was established according to Fried's physical frailty phenotype assessment and Rockwood and Mitnitski's Frailty Index, which is a strength of this study. Those are the two most common models of frailty, ${ }^{848}$ covering both conceptual definitions, ${ }^{12}$ used in most epidemiological studies ${ }^{1316}$ and recommended in clinical guidelines to assess frailty in older people. ${ }^{3} 49$ However, there are many other measurement instruments of frailty available, with much heterogeneity in their classification, measurement properties and predictive abilities, and the prevalence values of frailty will vary according to the conceptual model and measurement instrument. $^{846} 4849$

Fitness and frailty may be viewed as opposite ends of a challenging continuum, and that older people may be placed along that imaginary spectrum independently of age. ${ }^{50}$ According to this argumentation, the Frailty Index is not meant to be dichotomised into frail or non-frail. ${ }^{11}$ Rockwood et al have also proposed different Frailty Index cut-off points to define phenotypical population subgroups with increasing levels of frailty. For example, in one of their studies, ${ }^{51}$ they proposed $>0.21$ and $\geq 0.45$ as thresholds for the Frailty Index to assign individuals as frail or most frail, respectively. The prevalence of frailty varies according to the cut-off values defined for the Frailty Index. The figure in the online supplementary file 3 illustrates the distribution of the Frailty Index in the present sample and may help to better understand the prevalence of older people with frailty in the present sample along the fitness-frailty continuum. The observed data show the typical gamma distribution of the Frailty Index and the age independent limit to frailty of 0.7 reported consistently in previous studies. ${ }^{10} 32$

\section{Clinical implications}

This study shows that approximately one out of five older people prescribed for outpatient physiotherapy in an urban region in the western part of Germany has physical frailty and one out of three patients has frailty according to a broader concept of deficit accumulation. We assume that in most of these patients, a formal assessment and/ or recognition of frailty has not been performed prior to physiotherapy referral. Physiotherapists are in a favourable position to identify frailty, because they are often the first-line providers and clinical experts for treatment of frailty-associated functional impairments such as slowness, muscle weakness and low physical activity. ${ }^{22}$ But not only a formal screening or assessment can help to identify frailty. The common clinical presentations of frailty, such as falls, delirium and sudden immobility, can themselves also be used to alert healthcare professionals to the possible presence of frailty. ${ }^{3}$ Thus, physiotherapists working with older people should be aware of the high number of outpatients with frailty as well as the clinical presentations and formal measurement instruments to recognise and quantify frailty. Once frailty has been identified and quantified, the physiotherapist should inform the patient, the relatives and other healthcare professions involved in the older patient's healthcare about the patient's high vulnerability to negative health outcomes as well as the special healthcare needs required by this individual. $^{3452}$

A further clinical implication is that $\sim 20 \%-30 \%$ of older physiotherapy outpatients constitute a population of people with frailty who might benefit from additional physiotherapy led interventions. In clinical guidelines, ${ }^{3} 4952$ a progressive, individualised physical activity programme that contains a resistance training component has been recommended to treat the modifiable physical aspects of frailty, such as muscle weakness, functional impairments and inactivity. Such interventions should be designed as multicomponent exercise programmes, of long duration ( $\geq 5$ months), performed three times per week, and for $30-45$ min per session. ${ }^{53}$ In a recent umbrella review of seven systematic reviews on the effectiveness of exercise interventions on physical function in community-dwelling frail older people, the authors concluded that interventions for prefrail and frail older adults should include multicomponent exercises, including in particular resistance training, as well as aerobic, balance and flexibility tasks and that these interventions are effective for improving muscular strength, gait speed and physical performance. ${ }^{54}$

In the present sample, $28 \%$ and $17 \%$ of participants reported exhaustion (fatigue) and unintentional weight loss in the physical frailty phenotype assessment, respectively. According to the guideline by Dent $e t a l,{ }^{49}$ it is conditionally recommended that persons with frailty are screened for reversible causes of fatigue, such as sleep apnoea, depression, anaemia, hypotension and hypothyroidism. It is also recommended to screen for the reversible causes of unintentional weight loss. Physiotherapists should be alarmed if a patient reports such signs of frailty in a formal or informal assessment, anamnesis or conversation. These patients can be referred to the general practitioner or any other specialised healthcare professional to further assess and treat the causes of fatigue and weight loss in relation to the frailty status. 


\section{CONCLUSIONS}

This is the first study to describe the prevalence of frailty in older people attending outpatient physiotherapy care. In conclusion, the prevalence is higher than in community-dwelling older adults, but follows the same distribution patterns, with higher prevalence figures according to the model of deficit accumulation $(31 \%)$ than according to a model of a physical frailty phenotype (18\%), in females and with increased age. Aspects of physical frailty, such as slow gait speed and low muscle strength, are significant contributors of frailty that can be influenced by physiotherapy treatments. To better understand the prevalence of frailty among older people in physiotherapy care, more studies from different countries are needed.

Acknowledgements We thank all participants for their time and involvement. We would further like to thank the staff of the participating physiotherapy clinics in Bochum, namely: Gesundheitszentrum Höner, Ergo-Med Centrum, Physiotherapie Detlef Puls, Praxis für Physiotherapie 'Hand Drauf', Böhn Krankengymnastik, Zentrum Vitalis, Physiotherapie Labitzke, Praxis für Physiotherapie Michael Schumann, Physiotherapie Lindemann, Bewegungsstätte Physiotherapie und Prävention, Ruhrsportreha im Marien-Hospital Wattenscheid. We would like to acknowledge the support in data acquisition by Svenja Hansen.

Contributors Study concept and design: TB, CT and CG. Acquisition of data: CZ, $\mathrm{CB}, \mathrm{LH}, \mathrm{JR}$ and TR. Analysis of data, interpretation of data, drafting the manuscript: TB. Manuscript revision for important intellectual content: CT, CZ, CB, LH, JR, TR and CG. Final approval of the version to be published: all authors.

Funding This research was funded by an intramural grant from the Hochschule für Gesundheit Bochum (University of Applied Sciences).

Competing interests None declared.

Patient consent for publication Not required.

Ethics approval Approval was obtained from the Ethical Review Board of the German Confederation for Physiotherapy (registration number: 2015-07).

Provenance and peer review Not commissioned; externally peer reviewed.

Data sharing statement Data can be obtained from the corresponding author upon reasonable request.

Open access This is an open access article distributed in accordance with the Creative Commons Attribution Non Commercial (CC BY-NC 4.0) license, which permits others to distribute, remix, adapt, build upon this work non-commercially, and license their derivative works on different terms, provided the original work is properly cited, appropriate credit is given, any changes made indicated, and the use is non-commercial. See: http://creativecommons.org/licenses/by-nc/4.0/.

\section{REFERENCES}

1. Deutscher Verband für Physiotherapie. Zahlen Daten, Fakten aus berufsrelevanten Statistiken. 2016. https://www.physio-deutschland. de/fileadmin/data/bund/Dateien_oeffentlich/Beruf_und_Bildung/ Zahlen_Daten_Falten/Zahlen_Daten_Fakten.pdf (accessed 23 Aug 2017).

2. GKV-Spitzenverband. Anzahl der Heilmittelverordnungen der Gesetzlichen Krankenversicherung (GKV) nach Alter und Therapiebereich im Jahr 2016. 2016. https://de.statista.com/ statistik/daten/studie/247537/umfrage/anzahl-der-heilmittelverord nungen-der-gkv-nach-alter-und-therapiebereich/ (accessed 7 Mar 2018).

3. Turner G, Clegg A, British Geriatrics Society. Best practice guidelines for the management of frailty: a British Geriatrics Society, Age UK and Royal College of General Practitioners report. Age Ageing 2014;43:744-7.

4. Clegg A, Young J, lliffe S, et al. Frailty in elderly people. Lancet 2013;381:752-62.

5. Kojima G. Frailty as a predictor of future falls among communitydwelling older people: a systematic review and meta-analysis. J Am Med Dir Assoc 2015;16:1027-33.
6. Chang SF, Lin PL. Frail phenotype and mortality prediction: a systematic review and meta-analysis of prospective cohort studies. Int J Nurs Stud 2015;52:1362-74.

7. Morley JE, Vellas B, van Kan GA, van Abellan Kan G, et al. Frailty consensus: a call to action. J Am Med Dir Assoc 2013;14:392-7.

8. de Vries NM, Staal JB, van Ravensberg CD, et al. Outcome instruments to measure frailty: a systematic review. Ageing Res Rev 2011;10:104-14.

9. Fried LP, Tangen CM, Walston J, et al. Frailty in older adults: evidence for a phenotype. J Gerontol A Biol Sci Med Sci 2001;56:M146-M157.

10. Mitnitski AB, Mogilner AJ, Rockwood K. Accumulation of deficits as a proxy measure of aging. ScientificWorldJournal 2001;1:323-36.

11. Rockwood K, Andrew M, Mitnitski A. A comparison of two approaches to measuring frailty in elderly people. J Gerontol A Biol Sci Med Sci 2007;62:738-43.

12. Cesari M, Gambassi G, van Kan GA, et al. The frailty phenotype and the frailty index: different instruments for different purposes. Age Ageing 2014;43:10-12.

13. Shamliyan T, Talley KM, Ramakrishnan R, et al. Association of frailty with survival: a systematic literature review. Ageing Res Rev 2013;12:719-36.

14. Collard RM, Boter $\mathrm{H}$, Schoevers RA, et al. Prevalence of frailty in community-dwelling older persons: a systematic review. J Am Geriatr Soc 2012;60:1487-92.

15. Drey $\mathrm{M}$, Wehr $\mathrm{H}$, Wehr $\mathrm{G}$, et al. The frailty syndrome in general practitioner care: a pilot study. Z Gerontol Geriatr 2011;44:48-54.

16. Kojima G. Prevalence of frailty in nursing homes: a systematic review and meta-analysis. J Am Med Dir Assoc 2015;16:940-5.

17. Salvi F, Morichi V, Grilli A, et al. Screening for frailty in elderly emergency department patients by using the Identification of Seniors At Risk (ISAR). J Nutr Health Aging 2012;16:313-8.

18. Sepehri A, Beggs $T$, Hassan A, et al. The impact of frailty on outcomes after cardiac surgery: a systematic review. J Thorac Cardiovasc Surg 2014;148:3110-7.

19. Lin HS, Watts JN, Peel NM, et al. Frailty and post-operative outcomes in older surgical patients: a systematic review. BMC Geriatr 2016;16:157.

20. Kim DH, Kim CA, Placide S, et al. Preoperative frailty assessment and outcomes at 6 months or later in older adults undergoing cardiac surgical procedures: a systematic review. Ann Intern Med 2016;165:650-60.

21. Romero-Ortuno R. Frailty in primary care. Interdiscip Top Gerontol Geriatr 2015;41:85-94.

22. Gustavson AM, Falvey JR, Jankowski CM, et al. Public health impact of frailty: role of physical therapists. J Frailty Aging 2017;6:2-5.

23. von Elm E, Altman DG, Egger M, et al. The Strengthening the Reporting of Observational Studies in Epidemiology (STROBE) statement: guidelines for reporting observational studies. J Clin Epidemiol 2008;61:344-9.

24. Demographia. World Urban Areas: 13th Annual Edition: 2017: 04 2017. http://www.demographia.com/db-worldua.pdf (accessed 20 Aug 2017).

25. Stadt Bochum. Bevölkerung 2016. 2017. https://www.bochum.de/ C125708500379A31/vwContentByKey/W29GFAN9348BOCMDE\# par1 (Accessed 20 Aug 2017).

26. Fried LP, Ferrucci L, Darer J, et al. Untangling the concepts of disability, frailty, and comorbidity: implications for improved targeting and care. J Gerontol A Biol Sci Med Sci 2004;59:M255-M263.

27. Jokanovic N, Tan EC, Dooley MJ, et al. Prevalence and factors associated with polypharmacy in long-term care facilities: a systematic review. J Am Med Dir Assoc 2015;16:535.e1-535.e12.

28. Gnjidic D, Hilmer SN, Blyth FM, et al. Polypharmacy cutoff and outcomes: five or more medicines were used to identify communitydwelling older men at risk of different adverse outcomes. J Clin Epidemiol 2012;65:989-95.

29. Roberts HC, Denison HJ, Martin HJ, et al. A review of the measurement of grip strength in clinical and epidemiological studies: towards a standardised approach. Age Ageing 2011;40:423-9.

30. Taylor HL, Jacobs DR, Schucker B, et al. A questionnaire for the assessment of leisure time physical activities. J Chronic Dis 1978;31:741-55.

31. Braun T, Thiel C, Schulz R-J, et al. Diagnostik und Behandlung physischer Frailty. DMW - Deutsche Medizinische Wochenschrift 2017; 142:117-22.

32. Searle SD, Mitnitski A, Gahbauer EA, et al. A standard procedure for creating a frailty index. BMC Geriatr 2008;8:24.

33. Abner EL, Kryscio RJ, Caban-Holt AM, et al. Baseline subjective memory complaints associate with increased risk of incident dementia: the PREADVISE trial. J Prev Alzheimers Dis 2015;2:11-16. 
34. Hébert R, Durand PJ, Dubuc N, et al. Frail elderly patients. New model for integrated service delivery. Can Fam Physician 2003;49:992-7.

35. Clegg A, Rogers L, Young J. Diagnostic test accuracy of simple instruments for identifying frailty in community-dwelling older people: a systematic review. Age Ageing 2015;44:148-52.

36. Braun T, Grüneberg C, Thiel C. German translation, cross-cultural adaptation and diagnostic test accuracy of three frailty screening tools : PRISMA-7, FRAIL scale and Groningen Frailty Indicator. Z Gerontol Geriatr 2018;51:282-92.

37. Santos-Eggimann B, Cuénoud P, Spagnoli J, et al. Prevalence of frailty in middle-aged and older community-dwelling Europeans living in 10 countries. J Gerontol A Biol Sci Med Sci 2009;64:675-81.

38. Biritwum RB, Minicuci N, Yawson AE, et al. Prevalence of and factors associated with frailty and disability in older adults from China, Ghana, India, Mexico, Russia and South Africa. Maturitas 2016;91:8-18.

39. Fuchs J, Scheidt-Nave C, Gaertner B, et al. [Frailty in Germany: status and perspectives: Results from a workshop of the German Society for Epidemiology]. Z Gerontol Geriatr 2016;49:734-42.

40. Buttery AK, Busch MA, Gaertner B, et al. Prevalence and correlates of frailty among older adults: findings from the German health interview and examination survey. BMC Geriatr 2015;15:22.

41. Saum KU, Dieffenbach AK, Müller $\mathrm{H}$, et al. Frailty prevalence and 10 -year survival in community-dwelling older adults: results from the ESTHER cohort study. Eur J Epidemiol 2014;29:171-9.

42. Griffin DW, Harmon DC, Kennedy NM. Do patients with chronic low back pain have an altered level and/or pattern of physical activity compared to healthy individuals? A systematic review of the literature. Physiotherapy 2012;98:13-23.

43. Chen S, Honda T, Chen T, et al. Screening for frailty phenotype with objectively-measured physical activity in a west Japanese suburban community: evidence from the Sasaguri Genkimon Study. BMC Geriatr 2015;15:36.
44. Castell MV, Sánchez M, Julián R, et al. Frailty prevalence and slow walking speed in persons age 65 and older: implications for primary care. BMC Fam Pract 2013;14:86

45. Ricci NA, Pessoa GS, Ferriolli E, et al. Frailty and cardiovascular risk in community-dwelling elderly: a population-based study. Clin Interv Aging 2014;9:1677-85.

46. Theou O, Brothers TD, Mitnitski A, et al. Operationalization of frailty using eight commonly used scales and comparison of their ability to predict all-cause mortality. J Am Geriatr Soc 2013;61:1537-51.

47. Kojima G, Tanabe M. Frailty is Highly Prevalent and Associated with Vitamin D Deficiency in Male Nursing Home Residents. J Am Geriatr Soc 2016;64:e33-e35.

48. Apóstolo J, Cooke R, Bobrowicz-Campos E, et al. Predicting risk and outcomes for frail older adults: an umbrella review of frailty screening tools. JBI Database System Rev Implement Rep 2017;15:1154-208.

49. Dent E, Lien C, Lim WS, et al. The Asia-Pacific Clinical Practice Guidelines for the Management of Frailty. J Am Med Dir Assoc 2017:18:564-75.

50. Romero-Ortuno R, O'Shea D. Fitness and frailty: opposite ends of a challenging continuum! Will the end of age discrimination make frailty assessments an imperative? Age Ageing 2013;42:279-80.

51. Rockwood K, Song X, Mitnitski A. Changes in relative fitness and frailty across the adult lifespan: evidence from the Canadian National Population Health Survey. CMAJ 2011;183:E487-E494.

52. Fairhall $N$, Langron $C$, Sherrington $C$, et al. Treating frailty--a practical guide. BMC Med 2011;9:83.

53. Theou O, Stathokostas L, Roland KP, et al. The effectiveness of exercise interventions for the management of frailty: a systematic review. J Aging Res 2011;2011:1-19.

54. Jadczak AD, Makwana N, Luscombe-Marsh N, et al. Effectiveness of exercise interventions on physical function in community-dwelling frail older people: an umbrella review of systematic reviews. JBI Database System Rev Implement Rep 2018;16:752-75. 